\title{
EBSD Analysis of Plastic Strain for Austenitic Steels
}

\author{
Yohei Sakakibara $^{1 *}$, Keiji Kubushiro ${ }^{1}$, Kyohei Nomura ${ }^{1}$ and Satoshi Takahashi ${ }^{1}$ \\ ${ }^{1}$ IHI Corporation, 1, Shin-nakahara-Cho, Isogo-ku, Yokohama, Japan \\ *yohei_sakakibara@ihi.co.jp
}

Keywords: EBSD, Austenitic steel, Plastic strain

\begin{abstract}
Analysis of plastic deformation for 3types of austenitic stainless steels which have various grain sizes was performed. The 3 methods of calculation were applied to this analysis; Intra-granular Distortion ${ }^{(1)}$, Grain Orientation Spread and Kernel Average Misorientation. It was found that there were the good correlations between plastic strain and parameters by Intra-granular distortion or GOS, in spite of various grain sizes of the samples. There are also the correlations between plastic strain and result by KAM in each sample, however the KAM values increase with grain size decreasing.
\end{abstract}

\section{Results and Discussion}

We applied the Intra-granular distortion method to evaluate Orientation change data in each grains obtained by EBSD from each specimen. Figure 1 shows the results. Below the strains of $15 \%$, the misorientation increases monotonically with strain increasing and exhibits the near values for different specimens regardless of grain size and steel type. However, the dispersion in calculated values in the high strain range increases up to about $0.5^{\circ}$ depending on steel types; this is not the case in the low strain range.

Figure 2 shows the maps of GOS values for KA-SUS304 interuppted at various strain. The maps show that when the strain is 5\%, the colers of the GOS in each grains exhibit almost the same, so misorientations in each grains are expected also almost the same. On the other hand, as the strain increases to $10 \%$ and then to $15 \%$, the difference in the GOS values of each grains themselves become larger. A similar tendency was also observed in the other steel types. The relationships between the strain and the GOS values in the other steel types are shown in

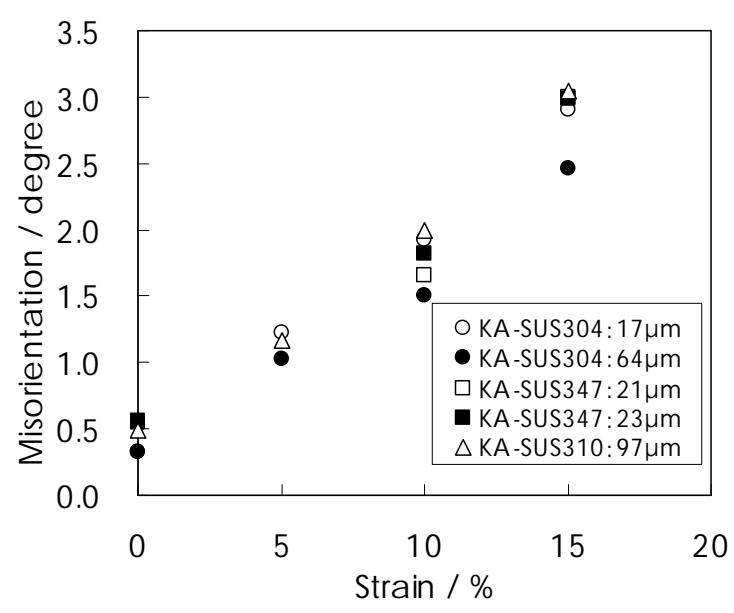

Fig.1 Plastic Strain dependence of misorientation calculated by Distortion for austenitic stainless steels figure 3. The GOS value is proportional to the strain and indicates almost the same value for different steel types, regardless of difference in grain size, steel type, and other measurement conditions. These results may suggest that the GOS values and misorientation obtained by the Intragranular distortion method are parameters that are independent of grain size and steel type with respect to the strain.

\section{References}

(1) YOSHIZAWA, M. NAKASHIRO, S. KIHARA, H. MAKI, Tetsu-to- Hagane, Vol.82， No.4， p61，

(1996)

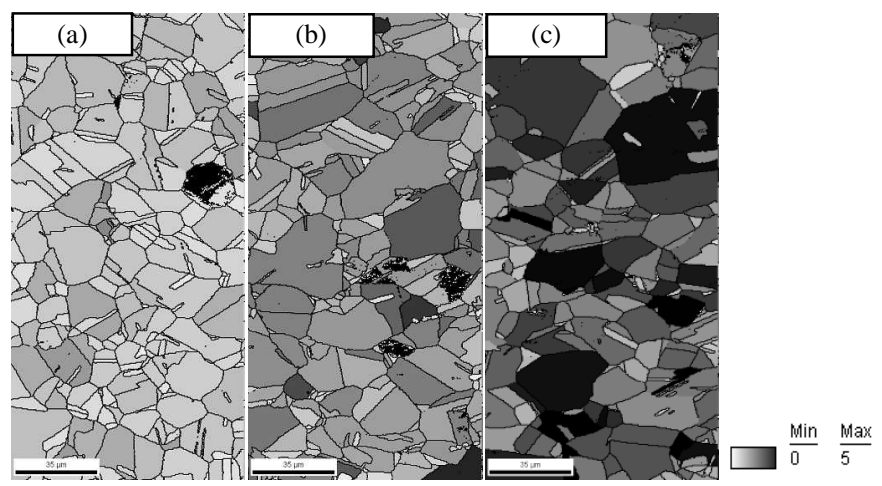

Fig.2 GOS maps of KA-SUS304 (a)Interrupted at $5 \%$, (b) at 10\%, (c) at $15 \%$

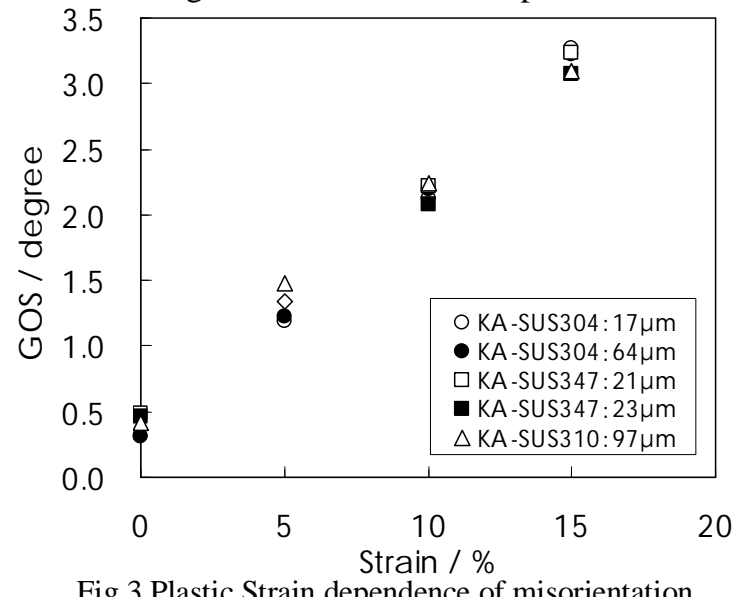

Fig.3 Plastic Strain dependence of misorientation calculated by GOS for austenitic stainless steels 